\title{
A Decline in Status 1 Listings: The Impact of Etiology and Medical Management of Acute Liver Failure
}

\section{SEE ARTICLE ON PAGE 1634}

In the current issue of Liver Transplantation, the outcomes of adult patients with acute liver failure (ALF) in the United States listed as status 1 for liver transplantation (LT) between 2002 and 2016 are presented. ${ }^{(1)}$ Key findings include the following: (1) black patients with ALF had higher Model for End-Stage Liver Disease scores and a lower likelihood of spontaneous recovery compared with whites with a larger proportion undergoing LT and (2) black patients had a lower 1-year survival after transplantation. These provocative findings confirm prior reports that wait-list mortality and post-LT outcomes vary by race and that the number of adults with ALF being referred for LT appears to have declined. ${ }^{(2,3)}$

\section{Liver Transplantation for ALF}

ALF is a rare clinical syndrome defined by the sudden onset of coagulopathy and mental status changes

Abbreviations: AIH, autoimmune hepatitis; ALF, acute liver failure; ALFSG, Acute Liver Failure Study Group; APAP, acetaminophen; $D I L I$, drug-induced liver injury; HAV, hepatitis $A$ virus; $H B V$, hepatitis $B$ virus; $L T$, liver transplantation.

Address reprint requests to Robert J. Fontana, M.D., Division of Gastroenterology and Hepatology, University of Michigan, 3912 Taubman Center, Ann Arbor, MI 48109. Telephone: 734-936-4780; FAX: 734-936-7392; E-mail: rfontana@med.umich.edu

Received September 10, 2019; accepted September 10, 2019.

Copyright (C) 2019 by the American Association for the Study of Liver Diseases.

View this article online at wileyonlinelibrary.com.

DOI $10.1002 / l t .25638$

Potential conflict of interest: Nothing to report. in a patient without known prior liver disease. Patients with ALF are at high risk for dying and currently account for $\sim 3 \%$ of the liver transplants in the United States. The Acute Liver Failure Study Group (ALFSG) is a multicenter National Institutes of Health network that has been prospectively studying the etiologies and outcomes of ALF since 1998. ${ }^{(2)}$ The ALFSG has demonstrated that patients with acetaminophen (APAP) overdose and hepatitis A virus (HAV)-related ALF have a much greater likelihood of transplant-free survival compared with patients with idiosyncratic drug-induced liver injury (DILI) and autoimmune hepatitis (AIH; Table 1). ${ }^{(2)}$ Furthermore, the proportion of ALFSG patients being listed as well as undergoing emergency LT significantly declined in the United States between 2002 and 2016 (Fig. 1). ${ }^{(2)}$ Whether these trends are due to more rapid diagnosis and treatment with effective therapies, such as $N$-acetylcysteine for APAP and non-APAP etiologies or less frequent use of blood products and mechanical ventilation, is unclear.

In addition, US Food and Drug Administration regulatory actions to reduce the amount of APAP in narcotic analgesic congeners and over-the-counter formulations may be having a favorable impact, but APAP hepatotoxicity remains the leading cause of ALF in the United States. An alternative explanation for the observed decline in status 1 listings could be improved categorization of patients as true ALF cases versus acute-on-chronic liver failure. Although 40\% of the status 1 patients were coded as "unknown" etiology by the United Network for Organ Sharing, it is unlikely that these patients truly had indeterminate ALF in light of recent studies that use formal causality assessment. ${ }^{(4)}$ Therefore, there is no convincing evidence that the etiologies nor incidence of ALF have substantially changed in the past 20 years in the United States. However, it remains plausible that the medical management of these critically ill patients is globally improving with fewer requiring transplant listing and undergoing LT. ${ }^{(2)}$ 
TABLE 1. Etiologies and Outcomes in Adult ALF Patients

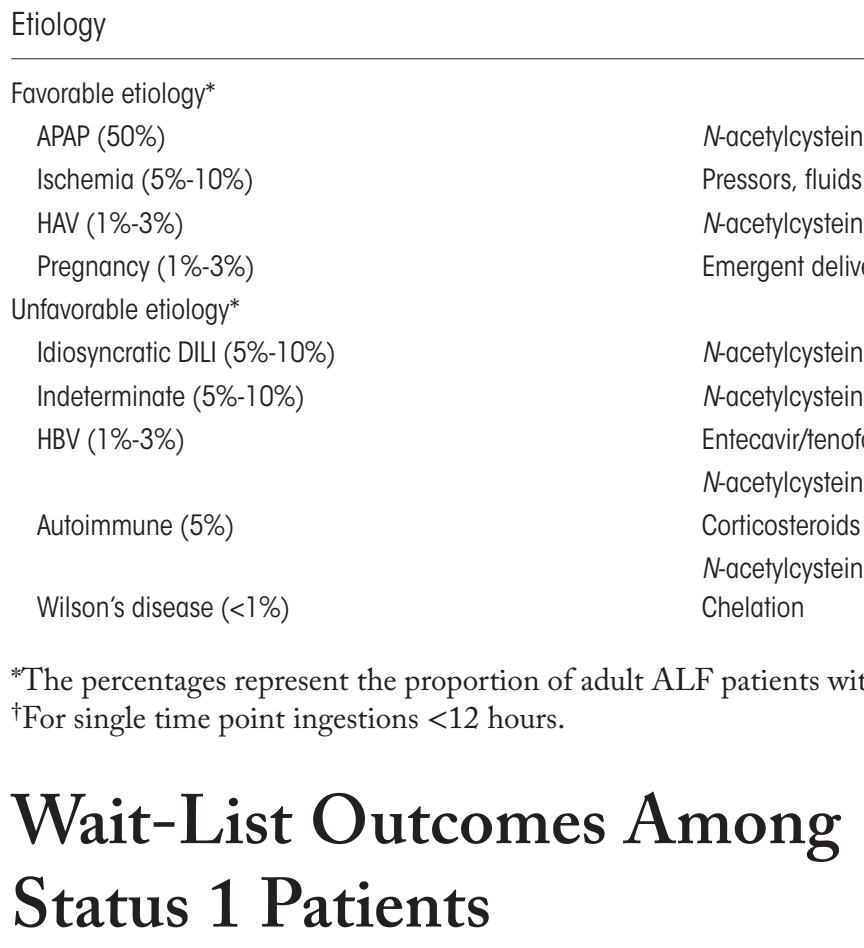

Although the proportion of ALF patients listed as status 1 has significantly declined over the past 15 years, black patients remain overrepresented compared with other racial groups. Furthermore, black ALF patients had a greater likelihood of dying or deteriorating prior to LT on univariate analysis but not when taking into account the competing risk of transplant. The greater need for LT in black ALF patients may, in part, be driven by their greater likelihood of having a diagnosis of DILI, AIH, or hepatitis $B$ virus $(\mathrm{HBV})$ that are all associated with a lower rate of spontaneous recovery compared with APAP overdose that predominates among white ALF patients. In support of this possibility, white ALF patients were also more likely to be removed from the waiting list due to clinical improvement.

The higher rate of LT among black ALF patients may also be related to racial differences in the likelihood of developing and presenting with severe DILI and AIH. The ALFSG showed that blacks were significantly more likely to present with idiosyncratic DILI compared with whites $(24.4 \%$ versus $14.9 \%, P=0.009) .{ }^{(5)}$ Recent European studies have also demonstrated that black patients with AIH present with more severe liver injury and are more likely to require transplant or die during follow-up. ${ }^{(6)}$ Prospective studies of idiosyncratic DILI have also suggested that black patients are more likely to have poorer clinical outcomes including
Recommended Treatment

Spontaneous Survival
$70 \%-80 \%$

$50 \%-60 \%$

$60 \%-70 \%$

$50 \%-60 \%$

$20 \%-30 \%$

$20 \%-30 \%$

$20 \%-30 \%$

$20 \%-30 \%$

$<5 \%$ higher short-term mortality compared with whites. ${ }^{(7,8)}$

The reasons for the poorer outcomes when controlling for disease etiology are unknown but could be mediated by genetic or immunological factors.

\section{Posttransplant Outcomes in Status 1 ALF Patients}

The 1-year survival of ALF transplant recipients significantly differed by patient race $(79.6 \%$ black versus $82.8 \%$ white and $89.3 \%$ Asian; $P=0.02$ ). The superior outcomes among Asian ALF LT recipients are similar to prior studies demonstrating better outcomes in Asians with chronic HBV undergoing LT compared with other groups. ${ }^{(9)}$ Although the median time to LT was similar across all racial groups at 2 days, the presence and severity of cerebral edema and infections at the time of LT were not available for analysis. Furthermore, other donor factors (age, cold ischemia time, and ABO compatibility) and recipient factors (age and use of induction immunosuppression) that may have impacted outcomes were also not available to review. ${ }^{(10)}$

Prior studies have demonstrated lower 1-year survival among black patients with cirrhosis undergoing LT compared with whites. ${ }^{(11)}$ Interestingly, donor-recipient race matching may be particularly important for black LT recipients with hepatocellular carcinoma and cirrhosis as has been shown for black recipients of kidney, lung, and heart transplant. ${ }^{(12,13)}$ However, it is unlikely that matching by donor race will be 


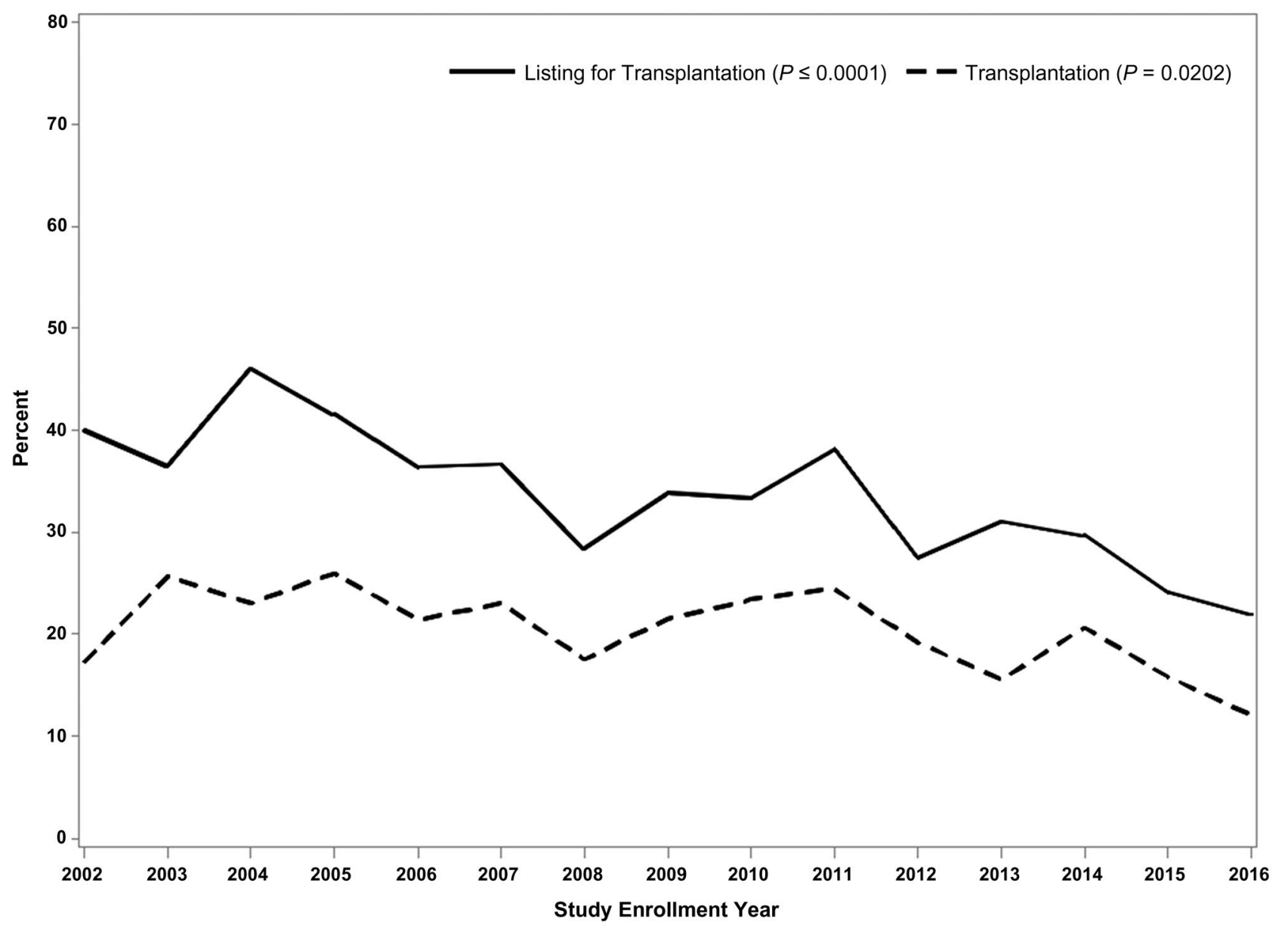

FIG. 1. Listing and receipt of liver transplantation in adult patients enrolled in the US ALFSG registry between 2002 and 2016 . The proportion of ALF patients being listed and undergoing emergency LT both significantly declined over time (by Cochran-Armitage test).

feasible under the emergency circumstances encountered in status $1 \mathrm{ALF}$ patients. Nonetheless, if an immunological basis for the differences in outcomes is demonstrated, immunosuppression protocols could be adapted in future race mismatched status 1 ALF transplant recipients.

In closing, Nephew et al. provide interesting and useful data regarding the incidence, etiologies, and outcomes of a large cohort of critically ill patients with a rare disease (ie, ALF) being cared for in more than 100 LT programs around the country. ${ }^{(1)}$ The significant decline in the listing of ALF patients as status 1 is good news for the general population and the many patients with cirrhosis awaiting LT. ${ }^{(2)}$ The higher rate of transplantation among black patients is likely due to their greater likelihood of having a nonrecoverable cause of ALF (ie, DILI or AIH) rather than being due to other clinical management issues or biases. Although 1-year survival after LT varied by patient race and ethnicity, the current analyses did not take into account other potential confounders that may have influenced outcomes. Additional prospective studies are now needed to identify modifiable recipient or donor factors that could lead to further improvements in ALF patient outcomes across all racial groups.

Robert J. Fontana, M.D. ${ }^{1}$

Valerie Durkalski, Ph.D. ${ }^{2}$

${ }^{1}$ Division of Gastroenterology and Hepatology

University of Michigan

Ann Arbor, MI

${ }^{2}$ Department of Public Health Sciences

Medical University of South Carolina

Charleston, SC 


\section{REFERENCES}

1) Nephew L, Zia Z, Ghabril M, Orman E, Lammert C, Chalasani $\mathrm{N}$. Black adult patients with acute liver failure are sicker and more likely to undergo liver transplantation than white patients. Liver Transpl 2019;25:1634-1641.

2) Reuben A, Tillman H, Fontana RJ, Davern T, McGuire B, Stravitz RT, et al. Outcomes in adults with acute liver failure between 1998 and 2013: an observational cohort study. Ann Intern Med 2016;164:724-732.

3) Germani G, Theocharidou E, Adam R, Karam V, Wendon J, O'Grady J, et al. Liver transplantation for acute liver failure in Europe: outcomes over 20 years from the ELTR database. J Hepatol 2012;57:288-296.

4) Ganger DR, Rule J, Rakela J, Bass N, Reuben A, Stravitz RT, et al.; for Acute Liver Failure Study Group. Acute liver failure of indeterminate etiology: a comprehensive systematic approach by an expert committee to establish causality. Am J Gastroenterol 2018;113:1319.

5) Forde KA, Reddy KR, Troxel AB, Sanders CM, Lee WM; for Acute Liver Failure Study Group. Racial and ethnic differences in presentation, etiology, and outcomes of acute liver failure in the United States. Clin Gastroenterol Hepatol 2009;7:11211126.

6) de Boer YS, Gerussi A, van den Brand FF, Wong GW, Halliday N, Liberal R, et al.; for Dutch Autoimmune Hepatitis Study Group. Association between black race and presentation and liver-related outcomes of patients with autoimmune hepatitis. Clin Gastroenterol Hepatol 2019;17:1616-1624.

7) Fontana RJ, Hayashi PH, Gu J, Reddy KR, Barnhart H, Watkins $\mathrm{PB}$, et al. Idiosyncratic drug-induced liver injury is associated with substantial morbidity and mortality within 6 months from onset. Gastroenterology 2014;147:96-108.

8) Chalasani N, Reddy KRK, Fontana RJ, Barnhart H, Gu J, Hayashi $\mathrm{PH}$, et al. Idiosyncratic drug induced liver injury in AfricanAmericans is associated with greater morbidity and mortality compared to Caucasians. Am J Gastroenterol 2017;112:1382-1388.

9) Thuluvath PJ, Guidinger MK, Fung JJ, Johnson LB, Rayhill SC, Pelletier SJ. Liver Transplantation in the United States, 19992008. Am J Transplant 2010;10(part 2):1003-1019.

10) Donnelly MC, Hayes PC, Simpson KJ. The changing face of liver transplantation for acute liver failure: assessment of current status and implications for future practice. Liver Transpl 2016;22:527-535.

11) Sarpel U, Suprun M, Sofianou A, Berger Y, Tedjasukmana A, Sekendiz Z, et al. Disentangling the effects of race and socioeconomic factors on liver transplantation rates for hepatocellular carcinoma. Clin Transpl 2016;30:714-721.

12) Silva JP, Maurina MN, Tsai S, Christians KK, Clarke CN, Mogal $\mathrm{H}$, et al. Effect of donor race matching on overall survival for African-American patients undergoing liver transplantation for hepatocellular carcinoma. J Am Coll Surg 2019;228:245-254.

13) Pang PS, Kamal A, Glenn JS. The effect of donor race on the survival of black Americans undergoing liver transplantation for chronic hepatitis C. Liver Transpl 2009;15:1126-1132. 